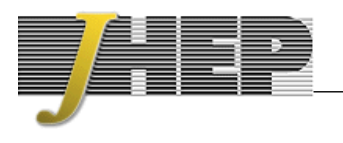

\title{
Decoupling of gravity on non-susy Dp branes
}

\author{
Kuntal Nayek and Shibaji Roy \\ Saha Institute of Nuclear Physics, \\ 1/AF Bidhannagar, Calcutta, 700064 India \\ E-mail: kuntal.nayek@saha.ac.in, shibaji.roy@saha.ac.in
}

ABSTRACT: We study the graviton scattering in the background of non-susy $\mathrm{D} p$ branes of type II string theories consisting of a metric, a dilaton and a $(p+1)$ form gauge field. We show numerically that in these backgrounds graviton experiences a scattering potential which takes the form of an infinite barrier in the low energy (near brane) limit for $p \leq 5$ and therefore is never able to reach the branes. This shows, contrary to what is known in the literature, that gravity indeed decouples from the non-susy $\mathrm{D} p$ branes for $p \leq 5$. For non-susy D6 brane, gravity couples as there is no such barrier for the potential. To give further credence to our claim we solve the scattering equation in some situation analytically and calculate the graviton absorption cross-sections on the non-susy branes and show that they vanish for $p \leq 4$ in the low energy limit. This shows, as in the case of BPS branes, that gravity does decouple for non-susy $\mathrm{D} p$ branes for $p \leq 4$ but it does not decouple for D6 brane as the potential here is always attractive. We argue for the non-susy D5 brane that depending on one of the parameters of the solution gravity either always decouples (unlike the BPS D5 brane) or it decouples when the energy of the graviton is below certain critical value, otherwise it couples, very similar to BPS D5 brane.

KEYwords: AdS-CFT Correspondence, D-branes, Superstring Vacua

ARXiv EPrint: 1506.08583 


\section{Contents}

1 Introduction 1

2 Non-susy $\mathrm{D} p$ branes and their BPS limits 3

3 Minimally coupled scalar scattering $\quad 5$

4 Graviton scattering: potentials $\quad 7$

5 Graviton scattering: absorption cross-section 10

6 Conclusion 14

\section{Introduction}

The AdS/CFT correspondence [1-4] is a holographic equivalence between certain quantum field theory without gravity and supergravity or string theory on a specific curved background. This was first conjectured by Maldacena [1] in the context of BPS D3-brane of type IIB string theory. The curved background in this case is the five-dimensional AdS space (apart from a 5-sphere) and the non-gravitational quantum field theory is the fourdimensional $\mathcal{N}=4$ super Yang-Mills theory which is a conformal field theory living on the boundary of $\mathrm{AdS}_{5}$. Thus the equivalence is holographic and it is also a strong-weak duality symmetry which is very useful to extract information about strongly coupled field theory from weakly coupled string (or supergravity) theory and vice-versa. String theory in the background of D3 brane consists of three parts: (i) theory living on the brane, (ii) theory living in the bulk and (iii) interaction between the two. It has been shown that there exists a decoupling (or a low energy) limit by which the interaction can be made to vanish and thus the field theory living on the brane gets decoupled from the bulk gravity theory. More precise way to see that the gravity decouples from the brane is to study the graviton scattering in the background of D3-brane [5-7]. Both from studying the scattering potential and also from the calculation of graviton absorption cross-section [5-10] one can see, that the scattering potential takes the form of an infinite barrier and the absorption cross-section vanishes in the decoupling limit. Therefore, a graviton propagating in the bulk and approaching the brane is never able to reach the brane in the decoupling limit and thus the bulk gravity gets decoupled from the brane.

The correspondence holds good even when there are less number of supersymmetries and with [11] or without conformal symmetries $[12,13]$. So, for example, BPS D $p$ branes (for $p \leq 5$ and $\neq 3$ ) of type II string theories has a decoupling limit in which the field theories on the brane get decoupled from the bulk gravity [14]. The field theories in these cases have 16 supercharges and no conformal symmetries giving rise to a non-AdS/non-CFT 
correspondence. One can explicitly check that decoupling of gravity on the brane indeed occurs by studying the graviton scattering on these branes. As in BPS D3 brane case, here also one can see that the graviton equation of motion takes the form of a Schrödingerlike equation, where the potential becomes infinite in the decoupling limit, indicating that gravity gets decoupled from the brane. One can further check the graviton absorption crosssection on the brane and indeed one finds that it vanishes in the decoupling limit which clearly indicates that the graviton does not reach the brane and the bulk gravity gets decoupled from the brane world-volume theory [15]. Decoupling of gravity does not occur for BPS D6 brane as the scattering potential in this case does not have a barrier. BPS D5 brane is a border-line between D6 brane and other $\mathrm{D} p$ branes. Here, the scattering potential indicates that gravity decouples if the energy carried by the graviton is below certain critical value, and above that value gravity couples to BPS D5 brane [15].

It is generally believed that AdS/CFT-like correspondence must hold good for more general backgrounds and even for the non-supersymmetric backgrounds. However, there is no explicit calculation in the literature showing the decoupling of gravity for the nonsupersymmetric gravitational systems. ${ }^{1}$ In this paper, we study the graviton scattering on the known non-supersymmetric (non-susy) D p-brane solutions of type II string theories [18, 19]. As a simple exercise we first look at the dynamics of a scalar coupled minimally to this background. We write the equation of motion in the string frame and show that the scalar satisfies, in this background, a Schrödinger-like equation with certain potential. The purpose of studying the minimally coupled scalar is that when we study the graviton scattering next, we will see that it will essentially (upto a multiplicative function) satisfy the same Schrödinger-like equation with the same potential in the background of non-susy $\mathrm{D} p$ brane solutions. We study the scattering potential numerically and show that in the decoupling limit the potentials act like an infinite barriers for the graviton to reach the brane for all $p \leq 5$. However, for $p=6$, there is no barrier for the potential and it always goes to negative infinity indicating that the gravity in this case does not decouple from the brane. To further strengthen our claim we compute the graviton absorption cross-sections for the non-susy $\mathrm{D} p$ branes. For this we need to solve the Schrödinger-like scattering equation. It is in general difficult to solve the equation, but, the equation can be solved both at the far region and at the near region and this is what is needed for obtaining the expression for the absorption cross-sections. However, unlike the BPS brane case [5, 6, 15] the near region solution for the non-susy case can be obtained only if the parameters of the solutions satisfy certain conditions discussed in section 4 . Then the solutions in the far region and the near region can be matched in the overlapping region and that fixes the arbitrary constant in the solution. ${ }^{2}$ From the form of the solution, we can straightforwardly obtain the expressions for the graviton absorption cross-sections on the brane. Then we show that the cross-sections vanish for all non-susy $\mathrm{D} p$ branes with $p \leq 4$ in the decoupling limit. We, therefore, conclude that the bulk gravity indeed decouples for all non-susy

\footnotetext{
${ }^{1}$ Some earlier comments and calculation about the non-decoupling of gravity for non-susy branes can be found in [16] and [17], however, we differ with their conclusion.

${ }^{2}$ Similar calculation has been done for BPS D3 brane in [5]. See also [8-10] for some earlier calculation of similar type for D1/D5 black holes.
} 
$\mathrm{D} p$ branes for $p \leq 4$. The calculation does not work for $p=5$, however, by studying the scattering potential we conclude that here depending on one of the parameters of the solution the decoupling occurs without any restrictions, otherwise, it occurs only when the energy of the graviton is below certain critical value, and above that value it couples. Even for $p=6$, the similar calculation does not go through and so, we conclude from the scattering potential that the gravity in this case couples as the potential here is attractive.

This paper is organized as follows. In the next section we briefly review the structure of non-susy D $p$ branes and their BPS limits. Then in section 3, we study the scattering of a minimally coupled scalar and obtain the form of the scattering potential. In section 4 , we study the graviton scattering and study the scattering potential numerically. In section 5 , the absorption cross-section of the graviton is obtained when the parameters of the solutions satisfy certain conditions. Finally we conclude in section 6 .

\section{Non-susy $\mathrm{D} p$ branes and their BPS limits}

In this section we briefly recall the non-susy $\mathrm{D} p$ brane solutions of type II string theories [19] and see how one can recover the BPS D $p$ brane solutions from them. The type II supergravity action we consider is given as,

$$
S=\frac{1}{16 \pi G_{10}} \int d^{10} x \sqrt{-g}\left[e^{-2 \phi}\left(R+4 \partial_{\mu} \phi \partial^{\mu} \phi\right)-\frac{1}{2 \cdot(8-p) !} F_{[8-p]}^{2}\right]
$$

where, $G_{10}$ is the ten dimensional Newton's constant, $g=\operatorname{det}\left(g_{\mu \nu}\right)$, with $g_{\mu \nu}$ being the ten dimensional string-frame metric and $R$ is its curvature scalar, $\phi$ is the dilaton and $F_{[8-p]}$ is an $(8-p) \mathrm{RR}$ form-field. One can solve the equations of motion following from this action with an appropriate form-field and a static, spherically symmetric $p$ brane metric (i.e., metric having the isometry $\operatorname{ISO}(p, 1) \times \mathrm{SO}(9-p)$ ) ansatz not respecting the supersymmetry condition and we get the non-susy $\mathrm{D} p$ brane solutions in the following form [19],

$$
\begin{aligned}
d s^{2}= & F(r)^{-\frac{1}{2}}\left(\frac{H(r)}{\tilde{H}(r)}\right)^{\frac{\delta}{2}}\left(-d t^{2}+\sum_{i=1}^{p}\left(d x^{i}\right)^{2}\right) \\
& +F(r)^{\frac{1}{2}}\left(\frac{H(r)}{\tilde{H}(r)}\right)^{\frac{\delta}{2}}(H(r) \tilde{H}(r))^{\frac{2}{7-p}}\left(d r^{2}+r^{2} d \Omega_{8-p}^{2}\right) \\
e^{2 \phi}= & F(r)^{\frac{3-p}{2}}\left(\frac{H(r)}{\tilde{H}(r)}\right)^{2 \delta}, \quad F_{[8-p]}=Q \operatorname{Vol}\left(\Omega_{8-p}\right) .
\end{aligned}
$$

The various functions appearing in the solution are defined as,

$$
\begin{aligned}
& H(r)=1+\frac{r_{p}^{7-p}}{r^{7-p}}, \quad \tilde{H}(r)=1-\frac{r_{p}^{7-p}}{r^{7-p}} \\
& F(r)=\left(\frac{H(r)}{\tilde{H}(r)}\right)^{\alpha} \cosh ^{2} \theta-\left(\frac{\tilde{H}(r)}{H(r)}\right)^{\beta} \sinh ^{2} \theta .
\end{aligned}
$$


In the above we have suppressed the string coupling constant $g_{\mathrm{s}}$ which is assumed to be small, $Q$ is the RR charge and $\operatorname{Vol}\left(\Omega_{8-p}\right)$ is the volume form of the transverse $(8-p)$ dimensional unit sphere. Also, $\alpha, \beta, \delta, \theta, Q$ and $r_{p}\left(r_{p}\right.$ has the dimension of length, but its actual form is different for different $p$ branes) are six parameters characterizing the solution. However, not all of them are independent. In fact, there are three relations among them following from the consistency of the equations of motion and they are,

$$
\begin{aligned}
\alpha-\beta & =\frac{p-3}{2} \delta \\
\frac{1}{2} \delta^{2}+\frac{1}{2} \alpha\left(\alpha-\frac{p-3}{2} \delta\right) & =\frac{8-p}{7-p} \\
Q & =(7-p) r_{p}^{7-p}(\alpha+\beta) \sinh 2 \theta .
\end{aligned}
$$

So, we can use these relations to eliminate three of the above six parameters and therefore, we take $\delta, \theta$ and $r_{p}$ as the independent parameters ${ }^{3}$ of the solution. In fact one can obtain $\alpha, \beta$ in terms of $\delta$ from the second relation in (2.4) as,

$$
\begin{aligned}
& \alpha=\sqrt{\frac{2(8-p)}{7-p}-\frac{(p+1)(7-p)}{16} \delta^{2}}+\frac{p-3}{4} \delta \\
& \beta=\sqrt{\frac{2(8-p)}{7-p}-\frac{(p+1)(7-p)}{16} \delta^{2}}-\frac{p-3}{4} \delta
\end{aligned}
$$

where for the reality of the parameters $\delta$ must be constrained as,

$$
|\delta| \leq \frac{4}{7-p} \sqrt{\frac{2(8-p)}{p+1}} .
$$

One may think that there are too many parameters in the solution which may violate Birkhoff's theorem. However, note that because of the form of $\tilde{H}(r)$ given in (2.3), the solution has a singularity at $r=r_{p}$, and therefore, Birkhoff's theorem does not apply here. So, $r>r_{p}$ is the physical region. We have also taken $(\alpha+\beta), \theta$ and $Q$ to be positive semidefinite without any loss of generality. Note that the non-susy $\mathrm{D} p$ brane solutions given in (2.2) is asymptotically flat and is magnetically charged. The corresponding electrically charged solutions can be obtained by first writing the metric in the Einstein frame using $g_{\mu \nu}^{\mathrm{E}}=e^{-\frac{\phi}{2}} g_{\mu \nu}$ and then making use of the transformation $g_{\mu \nu}^{\mathrm{E}} \rightarrow g_{\mu \nu}^{\mathrm{E}}, \phi \rightarrow-\phi$ and $F \rightarrow$ $e^{\frac{(3-p) \phi}{2}} * F$, where $*$ denotes the Hodge dual and finally going back to the string frame by the reverse metric transformation just given. For the electrically charged solution the gauge field takes the form,

$$
A_{[p+1]}=\frac{1}{2} \sinh 2 \theta\left(\frac{C(r)}{F(r)}\right) d t \wedge \cdots \wedge d x^{p}, \quad \text { with, } \quad C(r)=\left(\frac{H(r)}{\tilde{H}(r)}\right)^{\alpha}-\left(\frac{\tilde{H}(r)}{H(r)}\right)^{\beta}
$$

and $F(r)$ as given in (2.3). We will, however, work with the magnetically charged solutions.

\footnotetext{
${ }^{3}$ Attempts have been made to give physical meaning to these three parameters in terms of number of branes, number of anti-branes and a tachyon vev or tachyon parameter in [16, 20].
} 
Now to obtain the BPS $\mathrm{D} p$ brane from the non-susy $\mathrm{D} p$ brane solutions [19] given in (2.2), we notice that if we take $r_{p} \rightarrow 0$, then both $H(r)$ and $\tilde{H}(r)$ go to 1 , but the function

$$
F(r) \rightarrow 1+2\left(\alpha \cosh ^{2} \theta+\beta \sinh ^{2} \theta\right) \frac{r_{p}^{7-p}}{r^{7-p}} .
$$

Now if we further take $\theta \rightarrow \infty$ such that the product $2(\alpha+\beta) \sinh ^{2} \theta r_{p}^{7-p}=\bar{r}_{p}^{7-p}=$ fixed, then $F(r) \rightarrow \bar{H}(r)$, where $\bar{H}(r)=1+\bar{r}_{p}^{7-p} / r^{7-p}$ is the standard Harmonic function and the solution (2.2) reduces exactly to the standard BPS D $p$ brane solutions. Note that now all the extra paramaters from the non-susy $\mathrm{D} p$ brane solutions disappear and the BPS solutions are characterized by a single parameter $\bar{r}_{p}$ as expected.

In order to study the graviton scattering we first rewrite it in a more convenient form by introducing a new radial coordinate given by,

$$
r=\rho\left(\frac{1+\sqrt{G(\rho)}}{2}\right)^{\frac{2}{7-p}}, \quad \text { where, } \quad G(\rho)=1+\frac{4 r_{p}^{7-p}}{\rho^{7-p}} \equiv 1+\frac{\rho_{p}^{7-p}}{\rho^{7-p}} .
$$

In this new coordinate we have

$$
\begin{aligned}
H & =\frac{2 \sqrt{G(\rho)}}{1+\sqrt{G(\rho)}}, & \tilde{H}=\frac{2}{1+\sqrt{G(\rho)}} \\
d r^{2}+r^{2} d \Omega_{8-p}^{2} & =\left(\frac{1+\sqrt{G(\rho)}}{2}\right)^{\frac{4}{7-p}}\left[\frac{d \rho^{2}}{G(\rho)}+d \Omega_{8-p}^{2}\right] . &
\end{aligned}
$$

Using (2.10), we can rewrite the non-susy $\mathrm{D} p$ brane solution (2.2) as,

$$
\begin{aligned}
d s^{2} & =F(\rho)^{-\frac{1}{2}} G(\rho)^{\frac{\delta}{4}}\left(-d t^{2}+\sum_{i=1}^{p}\left(d x^{i}\right)^{2}\right)+F(\rho)^{\frac{1}{2}} G(\rho)^{\frac{1}{7-p}+\frac{\delta}{4}}\left(\frac{d \rho^{2}}{G(\rho)}+\rho^{2} d \Omega_{8-p}^{2}\right) \\
e^{2 \phi} & =F(\rho)^{\frac{3-p}{2}} G(\rho)^{\delta}, \quad F_{[8-p]}=Q \operatorname{Vol}\left(\Omega_{8-p}\right)
\end{aligned}
$$

where $G(\rho)$ is as given in $(2.9)$ and

$$
F(\rho)=G(\rho)^{\frac{\alpha}{2}} \cosh ^{2} \theta-G(\rho)^{-\frac{\beta}{2}} \sinh ^{2} \theta .
$$

The parameter relations remain exactly the same as given in (2.4). The BPS limit now would be given as $\rho_{p} \rightarrow 0, \theta \rightarrow \infty$, such that $(1 / 2)(\alpha+\beta) \rho_{p}^{7-p} \sinh ^{2} \theta=\bar{\rho}_{p}^{7-p}=$ fixed (where $\bar{\rho}_{p}^{7-p}=4 \bar{r}_{p}^{7-p}$ ). Note that in this case $G(\rho) \rightarrow 1$ and $F(\rho) \rightarrow \bar{H}(\rho)$, where $\bar{H}$ is the standard harmonic function of a BPS D $p$ brane and thus (2.11) reduces precisely to the BPS D $p$ brane solution. We will use the solution (2.11) to study both the minmally coupled scalar scattering and the graviton scattering in the following.

\section{Minimally coupled scalar scattering}

In this section we will study the scattering of a massless scalar $(\varphi)$ coupled minimally to the non-susy $\mathrm{D} p$ brane background and obtain the form of the scattering potential it 
experiences while moving in the background. The reason for studying this, as we will see, is that the graviton also experiences the same scattering potential (studied in the next section) as the scalar. The relevant part of the action in this case is

$$
S_{\text {scalar }}=\frac{1}{4 \pi G_{10}} \int d^{10} x \sqrt{-g} e^{-2 \phi} \partial_{\mu} \varphi \partial^{\mu} \varphi
$$

where $g_{\mu \nu}$ is the background metric in string frame and $\phi$ is the dilaton field. Note that we have omitted the Ricci scalar, the kinetic energy of the dilaton and the RR form-field terms from the action since they don't contribute to the scalar equation of motion. From (3.1) the scalar $\varphi$ is found to satisfy the following equation of motion,

$$
D_{\mu} \partial^{\mu} \varphi-2 D_{\mu} \phi \partial^{\mu} \varphi=0 .
$$

Now we assume that $\varphi$ has spherical symmetry in the transverse space (that means we are considering only scalar s-wave, since the higher partial waves will give an additional repulsive centrifugal term and will have greater chances of decoupling) and is independent of the spatial coordinates of the brane (assumed for simplicity). Thus $\varphi$ has the form,

$$
\varphi=\Phi(\rho) e^{i \omega t} .
$$

Now using (3.3), the scalar equation of motion (3.2) with the background given in (2.11) reduces to the following second order differential equation,

$$
\partial_{\rho}^{2} \Phi(\rho)+\left[\frac{8-p}{\rho}+\frac{\partial_{\rho} G(\rho)}{G(\rho)}\right] \partial_{\rho} \Phi(\rho)+\omega^{2} F(\rho) G(\rho)^{-\frac{6-p}{7-p}} \Phi(\rho)=0 .
$$

Redefining the radial coordinate as, $u=\omega \rho$ and introducing a function $g(u)$ by

$$
\Phi(u)=k(u) g(u), \quad \text { where, } \quad k(u)=\frac{1}{\sqrt{u^{8-p} G(u)}},
$$

(3.4) takes the form of a Schrödinger-like equation in terms of the new function as,

$$
\left(\partial_{u}^{2}-V(u)\right) g(u)=0
$$

where,

$$
V(u)=\frac{(8-p)(6-p)}{4 u^{2}}-\frac{1}{4}\left(\frac{\partial_{u} G(u)}{G(u)}\right)^{2}-F(u) G(u)^{-\frac{6-p}{7-p}}
$$

and

$$
G(u)=1+\frac{\left(\omega \rho_{p}\right)^{7-p}}{u^{7-p}}, \quad F(u)=G(u)^{\frac{\alpha}{2}} \cosh ^{2} \theta-G(u)^{-\frac{\beta}{2}} \sinh ^{2} \theta .
$$

Once we have the form of $g(u)$ by solving the equation (3.6), we can obtain the form of the scalar as

$$
\varphi(t, \rho)=\frac{1}{(\omega \rho)^{\frac{8-p}{2}}} \frac{g(\omega \rho)}{\sqrt{G(\omega \rho)}} e^{i \omega t} .
$$

Eq. (3.7) represents the scattering potentials the minimally coupled scalar experiences while moving in the background of non-susy $\mathrm{D} p$ branes. We will analyze these potentials (3.7) in the next section. 


\section{Graviton scattering: potentials}

In this section we will study the graviton scattering on the non-susy $\mathrm{D} p$ branes given in section 2. We will obtain the form of the scattering potential (which will have the same form (3.7) as the scattering potential of a minimally coupled scalar obtained in the previous section) and analyze it numerically. To study the scattering we need the linearized equations of motion from the action (2.1) in the background (2.11). The equations of motion can be linearized by perturbing the background metric as, $g_{\mu \nu}=\bar{g}_{\mu \nu}+h_{\mu \nu}$, where background metric is denoted with a 'bar'. The linearized forms of the dilaton and the graviton equations of motion are [15],

$$
\begin{aligned}
\Gamma(h)_{\mu \nu}^{\mu} \partial^{\nu} \phi+h^{\mu \nu} D_{\mu} \partial_{\nu} \phi= & -\frac{1}{4} \bar{R}_{\mu \nu} h^{\mu \nu}+\frac{1}{4}\left(D_{\mu} D_{\nu} h^{\mu \nu}-D^{2} h_{\mu}^{\mu}\right)+h^{\mu \nu} \partial_{\mu} \phi \partial_{\nu} \phi \\
& D_{(\mu} D_{\rho} h_{\nu)}^{\rho}-\frac{1}{2} D^{2} h_{\mu \nu}-\frac{1}{2} D_{\mu} D_{\nu} h_{\rho}^{\rho}+\bar{R}_{\rho(\mu} h_{\nu)}^{\rho}+\bar{R}_{\nu \rho \sigma \mu} h^{\rho \sigma} \\
= & 2 \Gamma(h)_{\mu \nu}^{\rho} \partial_{\rho} \phi-\frac{e^{2 \phi}}{2 \cdot(8-p) !}\left((8-p)(7-p) h^{\rho \sigma} F_{(\mu \mid \rho}^{\mu_{1} \cdots \mu_{6-p}} F_{\nu) \sigma \mu_{1} \cdots \mu_{6-p}}\right. \\
& \left.-\frac{8-p}{2} \bar{g}_{\mu \nu} h^{\rho \sigma} F_{\rho}^{\mu_{1} \cdots \mu_{7-p}} F_{\sigma \mu_{1} \cdots \mu_{7-p}}+\frac{1}{2} h_{\mu \nu} F^{2}\right) .
\end{aligned}
$$

Again, for the reasons given before for the scalar, we here consider only the graviton s-wave and assume (for simplicity) it to be independent of the spatial directions of the brane. So, the graviton takes the form,

$$
h_{\mu \nu}=\epsilon_{\mu \nu} h(\rho) e^{i \omega t}
$$

where $\epsilon_{\mu \nu}$ is the polarization tensor for the graviton. Further, we take gravitons to have the polarizations along the brane only and therefore, $\epsilon_{\mu \nu}=0$, for $\mu, \nu=p+1, p+2, \ldots, 9$. The transversality conditions of the graviton further restrict the polarization tensor, namely, we must have $\epsilon_{\mu 0}=0$ and for the consistency with (4.1) we take it to be traceless, i.e., $g^{a b} \epsilon_{a b}=0$, where $a, b=0,1, \ldots, p$. So, altogether there are $[p(p+1) / 2]-1$ number of possible choices of polarization tensor $\epsilon_{a b}$. Among them $p(p-1) / 2$ are off-diagonal and $p-1$ are diagonal. We choose the only non-zero off-diagonal components as $\epsilon_{12}=\epsilon_{21}=1$, and the only non-zero diagonal components as $\epsilon_{11}=-\epsilon_{22}=1$ satisfying all the restrictions on the polarization tensor. Since both types of polarizations give the same equation for $h(\rho)$, we give its form using (4.2) and (2.11) as,

$$
\begin{gathered}
\partial_{\rho}^{2} h(\rho)+\left[\frac{8-p}{\rho}+\frac{\partial_{\rho} F}{F}+\left(1-\frac{\delta}{2}\right) \frac{\partial_{\rho} G}{G}\right] \partial_{\rho} h(\rho) \\
+\left[\omega^{2} F G^{-\frac{6-p}{7-p}}+\frac{1}{4}\left(\frac{\partial_{\rho} F}{F}-\frac{\delta}{2} \frac{\partial_{\rho} G}{G}\right)^{2}-\frac{1}{2}\left(\frac{Q}{\rho^{8-p}}\right)^{2} F^{-2} G^{-\frac{3-p}{4} \delta-2}\right] h(\rho)=0 .
\end{gathered}
$$

Again redefining the radial coordinate by $u=\omega \rho$, and introducing the same function $g(u)$ as before, but now is related to $h(u)$ by

$$
h(u)=\tilde{k}(u) g(u), \quad \text { where }, \quad \tilde{k}(u)=\frac{1}{\sqrt{u^{8-p} F(u) G(u)^{1-\frac{\delta}{2}}}},
$$


we find, after some manipulation, that (4.4) reduces precisely to the same Schrödingerlike form in terms of $g(u)$ as (3.6) with the potential as given in (3.7). However, the graviton solution $h_{\mu \nu}(t, \rho)$, is obviously different (as can be seen from (4.5)) from that of the minimally coupled scalar (3.9). It is therefore clear that the graviton also experiences the same scattering potentials as a minimally coupled scalar when it moves in the background of non-susy $\mathrm{D} p$ branes.

We now take a close look at the graviton scattering potential $V(u)$ given in (3.7). First we note that it has three terms of which the first one is positive for $p \leq 5$ and is zero for $p=6$, and the second and third terms are always negative. So, the potential has both repulsive and attractive pieces for $p \leq 5$, but it is always (for all $u$ ) attractive for $p=6$. In fact this is the reason that gravity does not decouple from the non-susy D6 branes. However, for all $p$, when $u \rightarrow \infty$, i.e., in the far region $G(u)=1+\left(\omega \rho_{p}\right)^{7-p} / u^{7-p} \rightarrow 1$ and as a result $F(u)=G(u)^{\alpha / 2} \cosh ^{2} \theta-G(u)^{-\beta / 2} \sinh ^{2} \theta \rightarrow 1$ and so, we find from (3.7) that the potential $V(u) \rightarrow-1$. On the other hand in the near region, i.e., when $u \rightarrow 0$, $V(u) \rightarrow-1 /\left(4 u^{2}\right)-F(u) G(u)^{-(6-p) /(7-p)}$ and since $F(u)$ is positive and the second term is negative, $V(u) \rightarrow-\infty$. So, one might think that since in the near region the potential is attractive, gravity will not decouple from the brane. But this is not true. The point is that in between $u=\infty$ and $u=0$, there might exist some $u$, where the potential can have a maximum. We find that this is indeed the case, however, it is difficult to find the value of $u$ analytically where the maximum of the potential occurs because of its complicated form. So, we have plotted the potentials $V(u)$ given in (3.7) versus $u$ for different values of the parameters in figures 1,2 , and 3 . We have taken $\theta=0,{ }^{4}$ and $\delta=\delta_{c} / 2$, where $\delta_{c}=\frac{4}{7-p} \sqrt{\frac{2(8-p)}{p+1}}$ which is the maximum allowed value of $|\delta|$. These are some typical values of the parameters to see the variation of the potential $V(u)$. Actually we have seen that the variations of these two parameters have little effects on the potential. In figures 1, 2, and 3, we have taken $\omega \rho_{p}=1,0.1$, and 0.01 and then plotted $V(u)$ for various values of $p$. We notice that for $\omega \rho_{p}=1$, the potentials have maxima for each values of $p=1,2,3,4$ (also for $p=5$ but it is not visible because of the scale chosen and will be clear in figures 2,3) except for $p=6$. For $\omega \rho_{p}=0.1$, the maxima shift towards the origin and rises sharply for all values of $p$ except for $p=6$. When $\omega \rho_{p}=0.01$, the maxima of the potentials further shift towards the origin and take very large values for all values of $p$, but goes to large negative value for $p=6$. Note that as we scale down $\omega \rho_{p}$ by a factor of 10 , the potentials roughly rise by a factor of 100 . So, the potentials are approaching to infinity much faster than $\omega \rho_{p}$ going to zero. Thus eventually when we take $\omega \rho_{p} \rightarrow 0$, i.e., in the decoupling limit ${ }^{5}$ the potentials will rise to positive infinity near the origin for all values of $p$ except for $p=6$. For $p=6$ it will go to negative infinity without any maximum in between. Due to the infinite potential barriers, the graviton coming from infinity will not be able to

\footnotetext{
${ }^{4}$ This is taken for simplicity. However, note that by the relation (2.4), this implies that the brane is chargeless and this is a characteristic of a non-susy brane as a BPS brane should always be charged. Therefore, when we vary $\omega \rho_{p}$ in the plots figures $1,2,3$, the branes always remain non-susy even when we take the decoupling limit, namely, $\omega \rho_{p} \rightarrow 0$.

${ }^{5}$ Note that $\rho_{p} \sim \ell_{\mathrm{S}}$, where $\ell_{\mathrm{S}}$ is the fundamental string length and so, in the decoupling limit $\ell_{\mathrm{S}} \rightarrow 0$ implies $\rho_{p} \rightarrow 0 . \omega \rho_{p}$ is the dimensionless parameter which also tends to zero in the decoupling limit.
} 


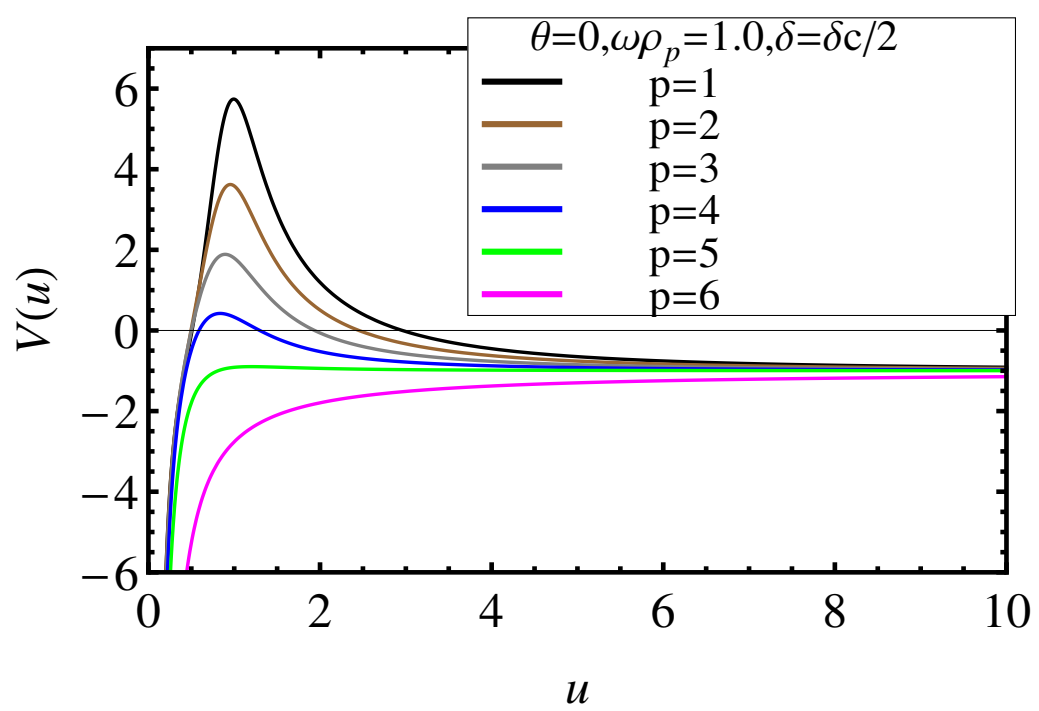

Figure 1. Plot of the potential $V(u)$ given in (3.7) vs $u$. Here the potential is given for $\theta=0$, $\omega \rho_{p}=1.0$ and $\delta=\delta_{c} / 2=\frac{2}{7-p} \sqrt{\frac{2(8-p)}{p+1}}$.

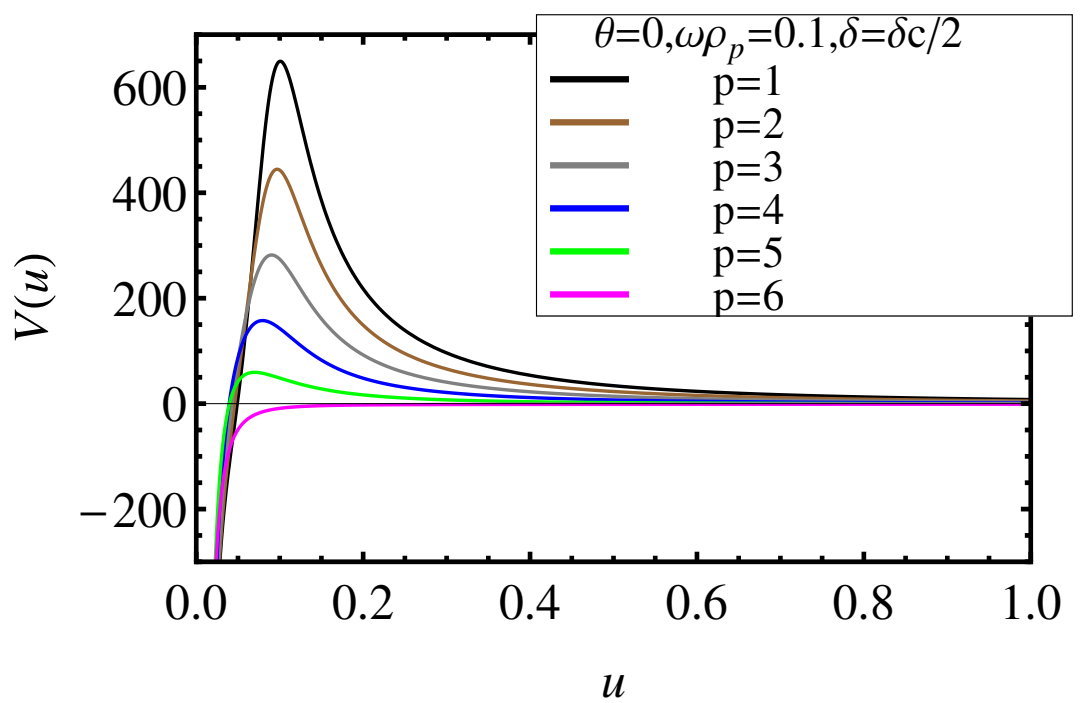

Figure 2. Plot of the potential $V(u)$ given in (3.7) vs $u$. Here the potential is given for $\theta=0$, $\omega \rho_{p}=0.1$ and $\delta=\delta_{c} / 2=\frac{2}{7-p} \sqrt{\frac{2(8-p)}{p+1}}$.

overcome them and reach the brane and therefore bulk gravity gets decoupled on all the non-susy $\mathrm{D} p$ branes for $p \leq 5$ and since for $p=6$, there is no potential barrier, the graviton will reach the brane and therefore gravity does not decouple from the non-susy D6 brane. We will discuss more on $p=5$ case towards the end of next section.

Now one can easily check that in the BPS limit $\left(\rho_{p} \rightarrow 0, \theta \rightarrow \infty\right.$, such that $(1 / 2)(\alpha+$ $\beta) \rho_{p}^{7-p} \sinh ^{2} \theta=Q /(7-p)=\bar{\rho}_{p}^{7-p}=$ fixed $), G(\rho) \rightarrow 1$ and $F(\rho) \rightarrow 1+Q /\left\{(7-p) \rho^{7-p}\right\}$, 


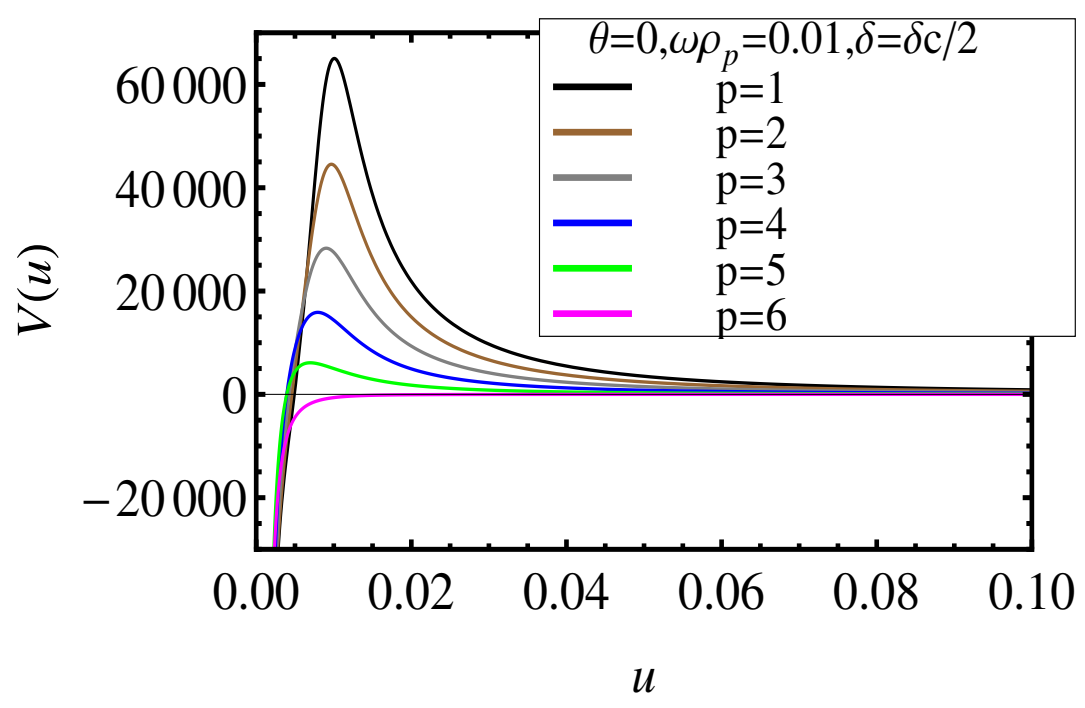

Figure 3. Plot of the potential $V(u)$ given in (3.7) vs $u$. Here the potential is given for $\theta=0$, $\omega \rho_{p}=0.01$ and $\delta=\delta_{c} / 2=\frac{2}{7-p} \sqrt{\frac{2(8-p)}{p+1}}$.

the standard harmonic function of a BPS $\mathrm{D} p$ brane, the potentials $V(u)$ for the non-susy $\mathrm{D} p$ branes as given in (3.7) reduce precisely to the scattering potentials of a graviton (or a minimally coupled scalar) for a BPS D $p$ branes given in eq. (4.6) of [15].

To further support our claim that bulk gravity does decouple from the non-susy $\mathrm{D} p$ brane world volumes we compute the graviton absorption cross-sections on the non-susy $\mathrm{D} p$ branes in the next section and show that they vanish in the decoupling limit.

\section{Graviton scattering: absorption cross-section}

To compute the graviton absorption cross section we will solve the Schrödinger-like scattering equation given in (3.6),

$$
\left(\partial_{u}^{2}-V(u)\right) g(u)=0, \quad \text { with, } \quad V(u)=\frac{(8-p)(6-p)}{4 u^{2}}-\frac{1}{4}\left(\frac{\partial_{u} G(u)}{G(u)}\right)^{2}-F(u) G(u)^{-\frac{6-p}{7-p}}
$$

where $G(u)$ and $F(u)$ are as given before in (3.8). It is difficult to solve this equation in general and so, as usual, we will solve it both in the far region and in the near region and then match the two solutions in the overlapping region to fix a constant in the solution. In the far region $u \gg \omega \rho_{p}$ and therefore $G(u) \approx 1$ and $F(u) \approx 1$ and then (5.1) reduces to

$$
\left[\partial_{u}^{2}+\left(1+\frac{1-4\left(\frac{7-p}{2}\right)^{2}}{4 u^{2}}\right)\right] g(u)=0 .
$$

This is the Bessel equation of order $\nu=\frac{7-p}{2}$ and so, the solution is,

$$
g(u)=c_{\infty} \sqrt{u} J_{\frac{7-p}{2}}(u)
$$


where $J(u)$ is the Bessel function and $c_{\infty}$ is a constant. Now with this solution the graviton takes the form (see (4.5))

$$
h(u)=\frac{g(u)}{\sqrt{u^{8-p} F(u) G(u)^{1-\frac{\delta}{2}}}}=\frac{c_{\infty} u^{-\frac{7-p}{2}} J_{\frac{7-p}{2}}(u)}{\sqrt{F(u) G(u)^{1-\frac{\delta}{2}}}} .
$$

Let us define another function by,

$$
\tilde{g}(u)=\frac{g(u)}{\sqrt{u^{8-p}}}, \quad \text { and so, } \quad h(u)=\frac{\tilde{g}(u)}{\sqrt{F(u) G(u)^{1-\frac{\delta}{2}}}}
$$

then the solution in the far region is

$$
\tilde{g}_{\infty}(u)=c_{\infty} u^{-\frac{7-p}{2}} J_{\frac{7-p}{2}}(u) .
$$

Now to find a solution in the near region we note that, unlike in the BPS case $[5,6,15]$, here it is difficult to get an analytic solution of the equation (5.1) in general for all $u$ in the near region. So, in the following we go over to certain region of space (within the near region) where we can solve (5.1) exactly and also have a matching in the overlapping region of the solution (5.6) in the far region and that in the near region. This is possible as we will see if the parameters of the solution are chosen appropriately. Now in the near region, we first make a coordinate transformation

$$
z=\frac{2}{5-p} \frac{\left(\omega \rho_{p}\right)^{\frac{7-p}{2}}}{u^{\frac{5-p}{2}}}, \quad \text { for, } \quad p<5
$$

and note that as long as $z \gg\left(\omega \rho_{p}\right)^{\frac{7-p}{2}}, u \ll 1$, i.e., we are in the near region where we will find a solution of eq. (5.1). The solution in the far region $\left(u \gg \omega \rho_{p}\right)$ and that in the near region $(u \ll 1)$ must be matched in the overlapping region to find the arbitrary constant $c_{\infty}$ and so, $\omega \rho_{p} \ll 1$ and this gives a restriction on the parameter $\rho_{p}$ of the non-susy $\mathrm{D} p$ brane solutions. We further impose the condition that $z \ll \omega \rho_{p}$. Note that when $\omega \rho_{p} \ll 1$, there is no contradiction of this with the previous condition $z \gg\left(\omega \rho_{p}\right)^{\frac{7-p}{2}}$. So, $z$ is in the range $\left(\omega \rho_{p}\right)^{\frac{7-p}{2}} \ll z \ll \omega \rho_{p}$. The scattering equation (5.1) then can be simplified as,

$$
\begin{gathered}
{\left[\partial_{z}^{2}+\left(\frac{1-4 \frac{(7-p)^{2}}{(5-p)^{2}}}{4 z^{2}}\right)+\left(\frac{\frac{2}{5-p}\left(\omega \rho_{p}\right)}{z}\right)^{\frac{2(7-p)}{5-p}}\right.} \\
\times\left\{1+\left(b^{2}-\frac{6-p}{7-p}\right)\left(\frac{z}{\frac{2}{5-p}\left(\omega \rho_{p}\right)}\right)^{\frac{2(7-p)}{5-p}}\right\} \hat{g}(z)=0
\end{gathered}
$$

where we have defined,

$$
b=\sqrt{\frac{\alpha}{2} \cosh ^{2} \theta+\frac{\beta}{2} \sinh ^{2} \theta}, \quad \text { and } \quad \hat{g}(z)=z^{\frac{7-p}{2(5-p)}} g(z) .
$$


Note from (5.8) that since $z \ll \omega \rho_{p}$, the first term in the curly bracket will dominate (assuming $b \sim \mathcal{O}(1)$ or $\ll 1$ ) and that will make the differential equation difficult to solve. On the other hand, if we further impose the condition on the parameter $b$ such that $z \gg b^{-\frac{5-p}{7-p}}\left(\omega \rho_{p}\right)$, then it is the $b^{2}$ term in the curly bracket which will dominate. We point out that this is possible if $b \gg 1$, implying that the parameter $\theta$ is large ${ }^{6}$ and since $\theta$ is an independent parameter of the solution both the conditions $z \gg\left(\omega \rho_{p}\right)^{\frac{7-p}{2}}$ and the previous one can be simultaneously satisfied. Now with these conditions (5.8) can be rewritten in the following form,

$$
\left[\partial_{\hat{z}}^{2}+\left(1+\frac{1-4 \frac{(7-p)^{2}}{(5-p)^{2}}}{4 \hat{z}^{2}}\right)\right] \hat{g}(\hat{z})=0 .
$$

Here the radial coordinate $\hat{z}$ is defined as $\hat{z}=b z$. Again we recognize (5.10) as the Bessel equation of order $\nu=\frac{7-p}{5-p}$ and since we are interested in incoming wave for the near region, the relevant solution is,

$$
\hat{g}(z)=i \sqrt{b} z^{\frac{1}{2}}\left(J_{\frac{7-p}{5-p}}(b z)+i N_{\frac{7-p}{5-p}}(b z)\right)
$$

where in the above $J$ denotes the Bessel function, $N$ denotes the Neumann function. The near solution $\tilde{g}_{0}(z)$ therefore takes the form,

$$
\tilde{g}_{0}(z)=i z^{\frac{7-p}{5-p}}\left(J_{\frac{7-p}{5-p}}(b z)+i N_{\frac{7-p}{5-p}}(b z)\right) .
$$

Now the two solutions (5.6) and (5.12) can be matched in the common region when $\omega \rho_{p} \ll 1$ and this determines the constant $c_{\infty}$ in terms of known constants as,

$$
c_{\infty}=\frac{1}{\pi} 2^{\frac{(7-p)^{2}}{2(5-p)}} b^{-\frac{7-p}{5-p}} \Gamma\left(\frac{7-p}{5-p}\right) \Gamma\left(\frac{9-p}{2}\right) .
$$

Therefore, we can write the solutions both in the near region $\left(u \ll 1\right.$ or $\left(\omega \rho_{p}\right)^{\frac{7-p}{2}} \ll z \ll$ $\omega \rho_{p}$ and $\left.z \gg b^{-\frac{5-p}{7-p}}\left(\omega \rho_{p}\right)\right)$ and in the far region $\left(u \gg \omega \rho_{p}\right)$ as follows,

$$
\begin{aligned}
\tilde{g}_{0}(z) & =i z^{\frac{7-p}{5-p}}\left(J_{\frac{7-p}{5-p}}(b z)+i N_{\frac{7-p}{5-p}}(b z)\right) \\
\tilde{g}_{\infty}(u) & =\frac{1}{\pi} 2^{\frac{(7-p)^{2}}{2(5-p)}} b^{-\frac{7-p}{5-p}} \Gamma\left(\frac{7-p}{5-p}\right) \Gamma\left(\frac{9-p}{2}\right) u^{-\frac{7-p}{2}} J_{\frac{7-p}{2}}(u) .
\end{aligned}
$$

Once we have the function $\tilde{g}(u)$, we can obtain the form of the graviton by using (5.5) and (4.3) as,

$$
h_{\mu \nu}=\epsilon_{\mu \nu} \frac{\tilde{g}(\omega \rho)}{\sqrt{F(\omega \rho) G(\omega \rho)^{1-\frac{\delta}{2}}}} e^{i \omega t} .
$$

\footnotetext{
${ }^{6}$ Note that when we plotted the potential in figures $1,2,3$ to show the decoupling we have taken $\theta=0$ for simplicity, but, we have checked that potential has very similar behavior even for large $\theta$. This shows that decoupling actually occurs for generic values of $\theta$, however, a closed form solution of the scattering equation is possible only for large value of $\theta$ satisfying the condition given above.
} 
The graviton flux can then be calculated from (5.15) using the standard definition as,

$$
F=i \int_{\rho=\rho_{S}} \sqrt{-\bar{g}} e^{-2 \bar{\phi}} \bar{g}^{\rho \rho}\left(\left(\partial_{\rho} h_{\mu \nu}^{*}\right) h^{\mu \nu}-h_{\mu \nu}^{*} \partial_{\rho} h^{\mu \nu}\right) d^{p+1} x d \Omega_{8-p} .
$$

The integral is over a constant surface of radius $\rho=\rho_{S}$. We use (5.15) with the solutions (5.14) for the incoming waves of the graviton in (5.16) to obtain the absorption cross-section as,

$$
\begin{aligned}
\sigma_{p}\left(b, \omega, \rho_{p}\right) & =\frac{(2 \pi)^{8-p}}{\omega^{8-p} \Omega_{8-p}}\left|\frac{F_{0}^{\text {in }}}{F_{\infty}^{\text {in }}}\right| \\
& =\frac{\pi^{\frac{11-p}{2}}\left(\frac{2}{5-p}\right)^{\frac{9-p}{5-p}}}{2^{\frac{4}{5-p}}\left[\Gamma\left(\frac{7-p}{5-p}\right)\right]^{2} \Gamma\left(\frac{9-p}{2}\right)} b^{\frac{2(7-p)}{5-p}} \omega^{\frac{9-p}{5-p}} \rho_{p}^{\frac{(7-p)^{2}}{5-p}}
\end{aligned}
$$

where $b$ is a particular combination of parameters of non-susy $\mathrm{D} p$ brane solutions defined in (5.9) and $\Omega_{d}=\frac{2 \pi^{\frac{d+1}{2}}}{\Gamma\left(\frac{d+1}{2}\right)}$ is the volume of a $d$-dimensional unit sphre. $F_{0}^{\text {in }}$ and $F_{\infty}^{\text {in }}$ are the graviton fluxes for the incoming waves in the near and the far regions respectively. We thus see that the absorption cross-sections (5.17) for the non-susy D $p$ branes depend on all the three parameters of the solutions, namely, $\rho_{p}, \theta$ and $\delta$ (through $b$ ) as expected. Now since $\rho_{p} \sim \ell_{\mathrm{s}}$, the absorption cross-sections in string units take the form,

$$
\frac{\sigma_{p}\left(b, \omega, \rho_{p}\right)}{\ell_{\mathrm{s}}^{8-p}} \sim\left(\omega \rho_{p}\right)^{\frac{9-p}{5-p}}
$$

which indeed vanish in the decoupling limit $\omega \rho_{p} \rightarrow 0$ as long as $p \leq 4$, showing that graviton does not reach the branes or it decouples for non-susy $\mathrm{D} p$ branes for $p \leq 4$. We will discuss $p=5,6$ cases separately. The formula of the absorption cross-sections for the BPS D $p$ branes can be recovered from (5.17) by the BPS limit we discussed before. The BPS limit is given as $\rho_{p} \rightarrow 0$ and $\theta \rightarrow \infty$ (or $b \rightarrow \infty$ ) such that the product $b^{2} \rho_{p}^{7-p}=\bar{\rho}_{p}^{7-p}=$ fixed. We note that in this limit $\sigma_{p}\left(b, \omega, \rho_{p}\right) \rightarrow \sigma_{p}^{\mathrm{BPS}}\left(\omega, \bar{\rho}_{p}\right)$, where $\sigma_{p}^{\mathrm{BPS}}$ is the absorption cross-section on the BPS D $p$ branes obtained before in [15]. ${ }^{7}$

For $p=5$, the coordinate transformation in the near region (5.7) does not work and so the same method of obtaining the absorption cross-section would be problematic. Anyway, we will look at the potential (5.1) and argue how the decoupling occurs in this case. The potential (5.1) for $p=5$ takes the form,

$$
V(u)=\frac{3}{4 u^{2}}-\frac{1}{4}\left(\frac{\partial_{u} G(u)}{G(u)}\right)^{2}-F(u) G(u)^{-\frac{1}{2}} .
$$

We simplify the potential in the near region $\omega \rho_{5} \ll u \ll 1$ (this is possible when $\omega \rho_{5} \ll 1$ ) as we have done for other $\mathrm{D} p$ brane cases before. Then the potential (5.19) takes the form,

$$
V(u)=\frac{3}{4 u^{2}}-\left[1+\left(b^{2}-\frac{1}{2}\right) \frac{\left(\omega \rho_{5}\right)^{2}}{u^{2}}\right]
$$

\footnotetext{
${ }^{7}$ We can compare the BPS results we obtain with those given in the table 1 of [15]. We find that there are some mismatch in the expressions of $\sigma_{p}$ for $p=1,2$. The numerical factors for $p=1$ we get is $\frac{\pi^{4}}{12}$ instead of $\frac{2 \pi^{4}}{3}$ and for $p=2$ the factor we get is $\frac{\pi^{2}(\Gamma(1 / 3))^{2}}{\sqrt[3]{3} 5}$ instead of $\frac{\pi^{3}(\Gamma(1 / 3))^{2}}{\sqrt[3]{3} 2^{2} 5}$. For $p=3,4$ the numerical factors match with our results.
} 
where $b$ is as defined in (5.9). If $b$ is $\mathcal{O}(1)$ or $\ll 1$, then the second term in the square bracket of (5.20) can be neglected as $u \gg \omega \rho_{5}$ and so, $V(u)=3 /\left(4 u^{2}\right)-1$. Also since $u \ll 1$, the first term dominates and eventually becomes infinite in the near region. So, the graviton won't be able to reach the brane surmounting this infinite barrier and there is decoupling of gravity. On the other hand when $\theta$ is such that $b \omega \rho_{5} \gg u$, then the potential becomes,

$$
V(u)=\frac{3}{4 u^{2}}-b^{2} \frac{\left(\omega \rho_{5}\right)^{2}}{u^{2}} .
$$

In this case, the potential acts as an infinite barrier as long as $\omega<\frac{\sqrt{3}}{2} \frac{1}{b \rho_{5}}$ and there is a decoupling and if $\omega>\frac{\sqrt{3}}{2} \frac{1}{b \rho_{5}}$, gravity couples to D5 brane. So, this gives a restriction on the energy of the incident graviton for the decoupling to occur. This situation is very similar to the BPS D5 brane discussed in [15].

For $p=6$, the scattering potential (5.1) takes the form

$$
V(u)=-\frac{1}{4}\left(\frac{\partial_{u} G(u)}{G(u)}\right)^{2}-F(u) .
$$

Now since both the terms here are negative, there is no maximum anywhere and it is a monotonically decreasing function as $u$ varies from $\infty$ to 0 without any barrier. Therefore, gravity will couple to non-susy D6 branes similar to BPS D6 branes.

We would like to remark that in obtaining the closed form expressions for the absorption cross-sections for the graviton we solved the scattering equation (5.1) analytically. However, in solving (5.1) we had to assume two conditions (i) $\omega \rho_{p} \ll 1$ (needed to have an overlapping region for the far and the near brane solutions) and also (ii) $b$ (or $\theta$ ) $\gg 1$ (needed to have a closed form solution of (5.8)). Note that these two conditions ${ }^{8}$ together imply that the non-susy $\mathrm{D} p$ brane solutions are near-BPS or near-extremal. So, our result for the absorption cross-sections (5.17) can be trusted only in this near-extremal case. When we are far away from the extremality point there can be large corrections to the absorption cross-sections. But since our numerical results suggest that the decoupling must occur even when we are far away from the extremality point, the corrections must vanish in the decoupling limit $\omega \rho_{p} \rightarrow 0$, which implies that the corrections must involve a positive power of $\omega \rho_{p}$.

\section{Conclusion}

To conclude, in this paper we have studied graviton scattering on the non-susy $\mathrm{D} p$ branes of type II string theories. We obtained the linearized equation of motion of the graviton in the

\footnotetext{
${ }^{8}$ One might think that these two conditions suggest that non-susy branes are becoming BPS branes in the decoupling limit and what we are obtaining is basically the decoupling of gravity for these BPS branes and not the non-susy branes. However, the reason why this is not so is because these two conditions are not correlated. Note that in order to get BPS branes we must take $b \rightarrow \infty$ and $\omega \rho_{p} \rightarrow 0$ simultaneously such that the product $b^{2}\left(\omega \rho_{p}\right)^{7-p}=\left(\omega \bar{\rho}_{p}\right)^{7-p}=$ finite. Here in our calculation, there is no relation between the two conditions and they are taken independently. So, when we take the decoupling limit $\omega \rho_{p} \rightarrow 0, b$ remains large but finite and therefore, the non-susy branes remain non-susy. But if we take $b$ also to infinity in the correlated way we just mentioned, then we recover graviton scattering cross-section result for the BPS brane as expected.
} 
background of non-susy $\mathrm{D} p$ branes. We have shown that both the minimally coupled scalar and the graviton essentially satisfy the same Schrödinger-like scattering equation and from there we identify the potential the minimally coupled scalar or the graviton experiences while moving in this background. For all non-susy $\mathrm{D} p$ brane backgrounds we found that far away from the branes the potentials go over to -1 , while near the branes they take the value $-\infty$ as in the case of BPS D $p$ branes. However, because of the complicated form of the potentials it is difficult to understand its behavior in between. So, we have studied them numerically and plotted the potentials $V(u)$ versus $u$ in figures $1,2,3$ for various values of $p$ in each figure. As the non-susy branes have three independent parameters $\rho_{p}, \theta$ and $\delta$, the potentials also depend on them. For simplicity we have set $\theta=0$ and $\delta=\delta_{c} / 2$, where $\delta_{c}$ is the maximum allowed value of $\delta$ described in section 2 and then plotted $V(u)$ for three different values of $\omega \rho_{p}$ (where $\omega$ is the energy of the graviton), namely, 1.0, 0.1 and 0.01 in the three figures mentioned above to show the behavior in the intermediate region. We found that there are maxima in the potentials near the origin for each value of $p \leq 5$, but the maximum is absent for $p=6$. When we lower the value of $\omega \rho_{p}$, the same feature remains, but the height of the maxima rise sharply and they shift more towards the origin. We thus concluded that when we take the decoupling limit $\omega \rho_{p} \rightarrow 0$, there will be infinite barriers close to the origin for all $p \leq 5$, but for $p=6$, there will be no barrier and the potential will decrease monotonically to $-\infty$. Thus the graviton will not be able to reach the brane for $p \leq 5$ and there will be decoupling of bulk gravity from the brane world volume. On the other hand, for $p=6$, since there is no barrier, graviton will couple to the brane.

To give further support to our claim, we tried to solve the graviton scattering equation for the non-susy $\mathrm{D} p$ branes. We found that we can write a closed form solution in the far region away from the branes, but it is difficult to solve the differential equation in the near region for all $u$. We found that a closed form solution in the near region is possible if we go to a certain range of space within the near region. To make this possible we found that the parameters of the solutions must satisfy the conditions (i) $\omega \rho_{p} \ll 1$ and (ii) $b$ (or $\theta) \gg 1$. We have emphasized that this does not mean that decoupling occurs only for large values of $b$. Decoupling occurs even for small values of $b$, but the closed form solutions is possible only if we use this condition. From these solutions we have computed the graviton absorption cross sections for the non-susy $\mathrm{D} p$ branes and found that they depend on the parameter $b$ as well as some positive powers of $\omega \rho_{p}$ for $p \leq 4$. Thus we found that in the decoupling limit $\omega \rho_{p} \rightarrow 0$, the graviton absorption cross-sections vanish for $p \leq 4$ and therefore gravity decouples. We have also compared our results with the BPS results obtained before in $[5,6,15]$. For $p=5$, we were not able to solve the equation even with the conditions mentioned above, but by analyzing the scattering potential we concluded that in this case if the parameter $b$ is $\mathcal{O}(1)$ or $\ll 1$, then gravity decouples for all energies of the graviton (unlike the BPS D5 brane), but if $b \gg 1$, then gravity decouples only if the energy of the graviton is below certain critical value, otherwise it couples, similar to BPS D5 brane. Even for $p=6$, we argued by analyzing the scattering potential that the gravity in this case couples to D6 brane as there is no barrier in the potential for the graviton. Thus, contrary to what is known in the literature $[16,17]$, we have shown 
here that there is a decoupling of gravity on the non-susy $\mathrm{D} p$ branes very similar to the BPS branes, modulo some differences for the case of D5 brane. We remarked that though the decoupling of gravity occurs in general for the non-susy $\mathrm{D} p$ branes the closed form expressions for the absorption cross-sections can only be obtained for the near-BPS or near-extremal point. The expressions derived in (5.17) can not be trusted far away from the extremality and there can be large corrections proportional to some positive power of $\omega \rho_{p}$.

So far we have not discussed anything about the boundary theory or the theory on the brane. It would be very interesting to understand this aspect in detail. However, we would like to mention that since we are dealing with non-susy branes, there may be open string tachyon $[21,22]$ living on the brane. When $\theta=0$, the charge of the non-susy branes vanish (see (2.4)) and the world volume theory in this case would be purely tachyonic field theory. But when $\theta \neq 0$, there are gauge fields on the brane and so, the theory in this case would be pure Yang-Mills theory coupled with tachyon. It can be checked that when $\alpha+\beta=2$ and $\theta$ is large, the gravity theories reduce to some deformations of the near horizon geometry of BPS D $p$ branes. Thus the world volume theories would be some perturbations of pure Yang-Mills theories in various dimensions without any tachyon field. We hope to come back on these issues along with others in future.

\section{Acknowledgments}

We would like to thank the anonymous referee for raising some questions which made us realize that some derivations we had given in the earlier version of this paper were not quite correct. We have made those corrections in this version although that does not affect the main results, the graviton scattering, of the paper.

Open Access. This article is distributed under the terms of the Creative Commons Attribution License (CC-BY 4.0), which permits any use, distribution and reproduction in any medium, provided the original author(s) and source are credited.

\section{References}

[1] J.M. Maldacena, The Large- $N$ limit of superconformal field theories and supergravity, Int. J. Theor. Phys. 38 (1999) 1113 [Adv. Theor. Math. Phys. 2 (1998) 231] [hep-th/9711200] [INSPIRE].

[2] E. Witten, Anti-de Sitter space and holography, Adv. Theor. Math. Phys. 2 (1998) 253 [hep-th/9802150] [INSPIRE].

[3] S.S. Gubser, I.R. Klebanov and A.M. Polyakov, Gauge theory correlators from noncritical string theory, Phys. Lett. B 428 (1998) 105 [hep-th/9802109] [INSPIRE].

[4] O. Aharony, S.S. Gubser, J.M. Maldacena, H. Ooguri and Y. Oz, Large-N field theories, string theory and gravity, Phys. Rept. 323 (2000) 183 [hep-th/9905111] [INSPIRE].

[5] I.R. Klebanov, World volume approach to absorption by nondilatonic branes, Nucl. Phys. B 496 (1997) 231 [hep-th/9702076] [INSPIRE]. 
[6] S.S. Gubser, I.R. Klebanov and A.A. Tseytlin, String theory and classical absorption by three-branes, Nucl. Phys. B 499 (1997) 217 [hep-th/9703040] [INSPIRE].

[7] S.S. Gubser and A. Hashimoto, Exact absorption probabilities for the D3-brane, Commun. Math. Phys. 203 (1999) 325 [hep-th/9805140] [INSPIRE].

[8] S.R. Das and S.D. Mathur, Comparing decay rates for black holes and D-branes, Nucl. Phys. B 478 (1996) 561 [hep-th/9606185] [INSPIRE].

[9] S.R. Das and S.D. Mathur, Interactions involving D-branes, Nucl. Phys. B 482 (1996) 153 [hep-th/9607149] [INSPIRE].

[10] S.R. Das, G.W. Gibbons and S.D. Mathur, Universality of low-energy absorption cross-sections for black holes, Phys. Rev. Lett. 78 (1997) 417 [hep-th/9609052] [INSPIRE].

[11] I.R. Klebanov and E. Witten, Superconformal field theory on three-branes at a Calabi-Yau singularity, Nucl. Phys. B 536 (1998) 199 [hep-th/9807080] [INSPIRE].

[12] I.R. Klebanov and A.A. Tseytlin, Gravity duals of supersymmetric $\mathrm{SU}(N) \times \mathrm{SU}(N+M)$ gauge theories, Nucl. Phys. B 578 (2000) 123 [hep-th/0002159] [INSPIRE].

[13] I.R. Klebanov and M.J. Strassler, Supergravity and a confining gauge theory: Duality cascades and $\chi S B$-resolution of naked singularities, JHEP 08 (2000) 052 [hep-th/0007191] [INSPIRE].

[14] N. Itzhaki, J.M. Maldacena, J. Sonnenschein and S. Yankielowicz, Supergravity and the large- $N$ limit of theories with sixteen supercharges, Phys. Rev. D 58 (1998) 046004 [hep-th/9802042] [INSPIRE].

[15] M. Alishahiha, H. Ita and Y. Oz, Graviton scattering on D6-branes with B fields, JHEP 06 (2000) 002 [hep-th/0004011] [INSPIRE].

[16] P. Brax, G. Mandal and Y. Oz, Supergravity description of nonBPS branes, Phys. Rev. D 63 (2001) 064008 [hep-th/0005242] [INSPIRE].

[17] P. Brax, Graviton absorption by nonBPS branes, Phys. Lett. B 506 (2001) 369 [hep-th/0101077] [INSPIRE].

[18] B. Zhou and C.-J. Zhu, The Complete black brane solutions in D-dimensional coupled gravity system, hep-th/9905146 [INSPIRE].

[19] J.X. Lu and S. Roy, Static, non-SUSY p-branes in diverse dimensions, JHEP 02 (2005) 001 [hep-th/0408242] [INSPIRE].

[20] J.X. Lu and S. Roy, Supergravity approach to tachyon condensation on the brane- anti-brane system, Phys. Lett. B 599 (2004) 313 [hep-th/0403147] [INSPIRE].

[21] A. Sen, NonBPS states and Branes in string theory, hep-th/9904207 [INSPIRE].

[22] A. Sen, Tachyon dynamics in open string theory, Int. J. Mod. Phys. A 20 (2005) 5513 [hep-th/0410103] [INSPIRE]. 